\title{
Performance Assurance Program Plan
}

Prepared for the U.S. Department of Energy Assistant Secretary for Environmental Management 


\section{LEGAL DISCLAIMER}

This report was prepared as an account of work sponsored by an agency of the United States Government. Neither the United States Government nor any agency thereof, nor any of their employees, nor any of theit contractors, subcontractors or their employees, makes any warranty, express or implied, or assumes any legal liability or responsibility for the accuracy, completeness, or any third party's use of the results of such use of any information, apparatus, product, or process disclosed, or represents that its use would not infringe privately owned rights. Reference herein to any specific commercial product, process, or senice by trade name, ttademark, manufacturer, or otherwise; does not necessarily constitute or imply its endorsement, recommendation, or favoring by the United States Government or any agency thereof or its contractors or subcontractors. The views and opinions of authors expressed herein do not necessarily state or reflect those of the United States Government or any agency thereof.

This report has been reproduced from the best available copy. Available in paper copy and microfiche.

Available to the U.S. Department of Energy and its contractors from

U.S. Department of Energy

Office of Scientific and Technical Information (OSTI)

P.O. Box 62

Oak Fidge, TN 37831

(615) 576.8401

Available to the public from the U.S. Department of Commerce National Technical lnformation Service (NTIS)

5285 Port Royal Road

Springfield, VA 22161

(703) $487-4650$

Printed in the United States of Americe.

DISCLM-1.CHP (8-95) 


\section{Performance Assurance Program Plan}

\section{B. H. Rogers}

B\&W Protec, Inc., for

Fluor Daniel Hanford, Inc.

Date Published

August 1997

Prepared for the U.S. Department of Energy

Assistant Secretary for Environmental Management

DUES INOT CONTAIN CLASSIFIED OK UNCLASSIFIED CONTROLLED NUCLEAR INFORMATION

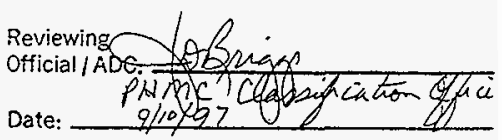

Approved for public release; distribution is unlimited 


\section{RELEASE AUTHORIZATION}

Document Number:
HNF-EP-0927

\section{Document}

Title:

\section{This document, reviewed in accordance with DOE Order 1430.1D, "Scientific and Technical Information Management," and DOE G 1430.1D-1, "Guide to the Management of Scientific and Technical Information," does not contain classified or sensitive unclassified information and is:}

\section{APPROVED FOR PUBLIC RELEASE}

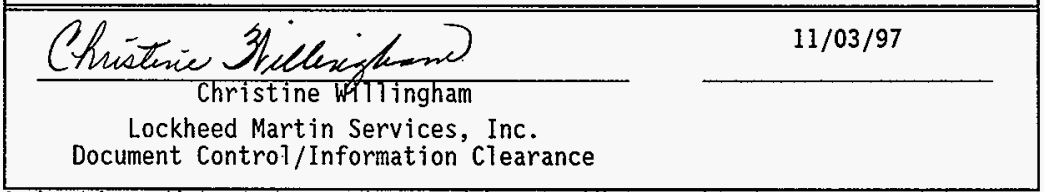

Reviewed for Applied Technology, Business Sensitive, Classified, Copyrighted, Export Controlled, Patent, Personal/Private, Proprietary, Protected CRADA, Trademark, Unclassified Controlled Huclear Information.

LEGAL. DISCLAIMER. This report was prepared as an account of work sponsored by an agency of the United States Government. Neither the United States Government nor any agency thereof, not any of their employees, nor any of their contractors, subcontractors or their employees, makes any warranty, express or implied, or assumes any legal liability or responsibility for the accuracy, completeness, or any third party's use or the results of such use of any information, apparatus, product, or process disclosed, or represents that its use would not infringe privately owned rights. Reference herein to any specific comercial product, process, or service by trade name, trademark, manufacturer, or otherwise, does not necessarily constitute or imply its endorsement, recomendation, or favoring by the United States Government or any agency thereof or its contractors or subcontractors. The views and opinions of authors expressed herein do not necessarily state or reflect those of the United States Government or any agency thereof. This report has been reproduced from the best available copy. Printed in the United States of America. 


\section{HNE-EP-0927}

Release Authorization Form 
HNF-EP-0927

This page intentionally left blank. 


\section{CONTENTS}

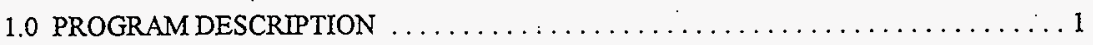

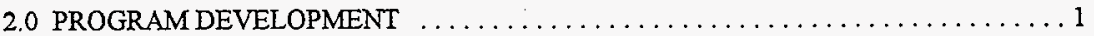

3.0 FUNCTIONS THAT COMPRISE PERFORMANCE ASSURANCE $\ldots \ldots \ldots \ldots \ldots .2$

4.0 METHODOLOGY FOR INTEGRATING PERFORMANCE ASSURANCE

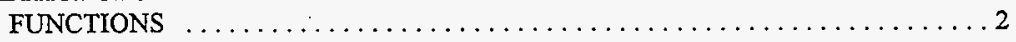

4.1 ORGANIZATIONAL RESPONSIBLITIES $\ldots \ldots \ldots \ldots \ldots \ldots \ldots \ldots \ldots \ldots \ldots \ldots$

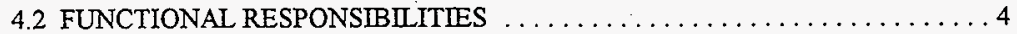

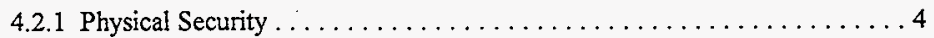

4.2.2 Hanford Patrol Training Academy $\ldots \ldots \ldots \ldots \ldots \ldots \ldots \ldots \ldots 10$

4.2 .3 Hanford Patrol .............................. 10

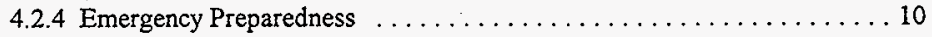

4.2.5 Risk Analysis \& Assessments . . . . . . . . . . . . . . . 10

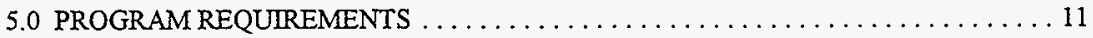

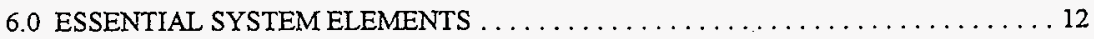

7.0 TEST DOCUMENTATION REQUIREMENTS $\ldots \ldots \ldots \ldots \ldots \ldots \ldots \ldots \ldots \ldots \ldots \ldots \ldots$

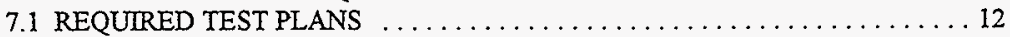

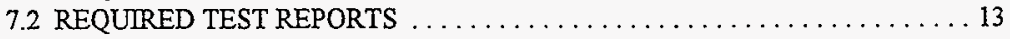

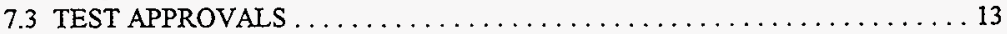

8.0 CORRECTIVE ACTION REQUIREMENTS $\ldots \ldots \ldots \ldots \ldots \ldots \ldots \ldots \ldots \ldots$

8.1 DEFICIENCIES IDENTIFIED DURING PERFORMANCE TESTING $\ldots \ldots \ldots 13$

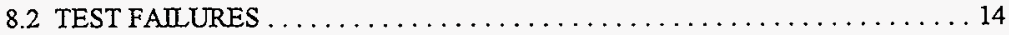

9.0 RESOURCES ................................... 14

9.1 PHYSICAL SECURITY AND SECURITY ENGINEERING AND

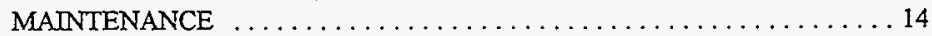

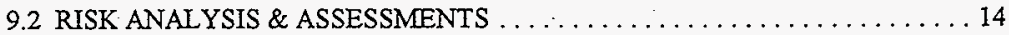

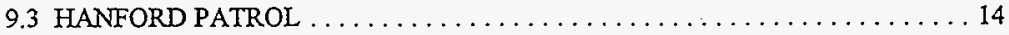

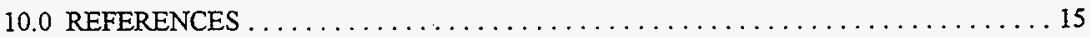

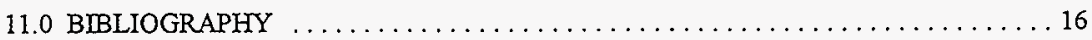

APPENDIX A: EXAMPLE - PERFORMANCE TEST PLAN $\ldots \ldots \ldots \ldots \ldots \ldots \ldots$ A-1 APPENDIX B: PERFORMANCE TEST REPORT OUTLINE $\ldots \ldots \ldots \ldots \ldots \ldots \ldots$ B-1

APPENDIX C: COMMAND AND CONTROL $\ldots \ldots \ldots \ldots \ldots \ldots \ldots \ldots \ldots \ldots \ldots \ldots \ldots \ldots \ldots$

APPENDIX D: DATA COLLECTION SHEET $\ldots \ldots \ldots \ldots \ldots \ldots \ldots \ldots \ldots \ldots \ldots \ldots \ldots \ldots$ 


\section{HNF-EP-0927}

\section{LIST OF TABLES}

1 Essential System Element Identification and Testing 5 


\section{PERFORMANCE ASSURANCE PROGRAM PLAN}

\subsection{PROGRAM DESCRIPTION}

B\&W Protec, Inc. (BWP) is responsible for implementing the Performance Assurance Program for the Project Hanford Management Contract (PHMC) in accordance with DOE Order 470.1, Safeguards and Security Program (DOE 1995a).

The Performance Assurance Program applies to safeguards and security (SAS) systems and their essential components (equipment, hardware, administrative procedures, Protective Force personnel, and other personnel) in direct support of Category I and II special nuclear material (SNM) protection.

Performance assurance includes several Hanford Site activities that conduct performance, acceptance, operability, effectiveness, and validation tests. These activities encompass areas of training, exercises, quality assurance, conduct of operations, total quality management, selfassessment, classified matter protection and control, emergency preparedness, and corrective actions tracking and trending.

The objective of the Performance Assurance Program is to capture the critical data of the tests, training, etc., in a cost-effective, manageable program that reflects the overall effectiveness of the program while minimizing operational impacts. To aid in achieving this objective, BWP will coordinate the Performance Assurance Program for Fluor Daniel Hanford, Inc. (FDH) and serve as the centrai point for data collection.

\subsection{PROGRAM DEVELOPMENT}

To develop a cost-effective approach to performance assurance, the U.S. Department of Energy (DOE) issued basic guidance to Operations Offices and contractor organizations. The guidance allows organizations to take credit for all the performance assurance activities being performed without any major changes in the conduct of operations. The improvements in the program will ensure an audit trail of performance assurance activities, and require documented corrective actions for unsatisfactory test results and formal tracking of these corrective actions until completion. This will not involve any organizational changes, but rather improve ways of documenting and managing the results of existing excellent programs.

The following process was used to develop the BWP Performance Assurance Program:

- Identified performance assurance functions

- Outlined methodologies for integrating the performance assurance functions

- Specified Performance Assurance Program requirements 
HNF-EP-0927

- Identified essential components associated with performance assurance along with the frequency of testing required

- Outlined test documentation requirements

- Described corrective action management requirements

- Specified resource requirements to support the Performance Assurance Program

- Provided an example of a performance test plan (Appendix A) and a test report format (Appendix B).

\subsection{FUNCTIONS THAT COMPRISE PERFORMANCE ASSURANCE}

The following functions comprise performance assurance:

- Security engineering and maintenance

- Security system testing

- Protective Force field training exercises

- Vulnerability analysis validation performance testing

- Alarm response and assessment performance testing

- Command post exercise

- Command field exercise

- Joint training exercise

- Limited-scope performance testing (LSPT)

- Material control and accountability (MC\&A) performance testing

- Personnel Security Assurance Program

- Internal assessments

- Corrective action management and tracking

- Emergency preparedness exercises.

\subsection{METHODOLOGY FOR INTEGRATING PERFORMANCE ASSURANCE FUNCTIONS}

\subsection{ORGANIZATIONAL RESPONSEILITIES}

This section discusses responsibilities for the following organizations:

- U.S. Department of Energy, Richland Operations Office (RI) SAS

- FDHProgram Management

- FDH Infrastructure \& Security 


\section{HNF-EP-0927}

- BWP

- PHMC subcontractors and enterprise companies.

RL SAS shall perform the following activities.

- Approve the FDH performance assurance plan.

- Ensure appropriate funding and milestones have been identified to accomplish FDH performance assurance activities.

- Observe performance assurance tests that encompass protection systems associated with a comprehensive site or facility threat scenario that demonstrates the overall facility SAS system effectiveness.

- Review performance assurance test reports that encompass a comprehensive site or facility threat scenario.

- Ensure corrective actions are identified for deficiencies and appropriately tracked by FDH.

FDH Program Management shall perform the following activities.

- Coordinate allocation of funds to support SAS performance assurance activities.

- Approve scheduling of performance assurance tests that encompass comprehensive threat scenarios in their respective facility(ies).

- Ensure appropriate corrective actions are identified for deficiencies, appropriately tracked, and ultimately resolved.

FDH Infrastructure \& Security shall perform the following activities.

- Designate BWP as the organization responsible for administration and oversight of the SAS Performance Assurance Program.

- Ensure appropriate funding is available to accomplish SAS performance assurance activities.

- Establish achievable milestones for completion of SAS performance assurance activities.

- Provide the SAS performance test schedule to RL SAS for approval by September 30 of each year.

- Ensure all SAS performance assurance activities are performed with the highest regard for the safety and health of all personnel. 
HNF-EP-0927

- Ensure appropriate corrective actions are identified for deficiencies, appropriately tracked, and ultimately resolved.

- Provide RL SAS with copies of performance assurance test reports that encompass a comprehensive site or facility threat scenario.

PHMC subcontractors and enterprise companies shall perform the following activities.

- Approve scheduling of performance tests involving comprehensive threat scenarios of their facility(ies).

- Include BWP in the planning and execution of any effectiveness tests affecting facilities containing Category I or II SNM.

- Implement appropriate correction planning to resolve identified problems or deficiencies.

BWP shall perform the following activities.

- Appoint a performance assurance point of contact responsible for administration and oversight of its Performance Assurance Program.

- Ensure the fiscal year SAS program plan reflects appropriate funding and milestones to accomplish performance assurance activities.

- Provide an annual performance test schedule to FDH.

- Update the performance assurance plan as required.

\subsection{FUNCTIONAL RESPONSIBLITIES}

Methodologies used to conduct required tests are outlined in the following subsections. These methodologies will not change; however, some additional reporting may be required to ensure an audit trail is established for results requiring corrective action plans.

\subsubsection{Physical Security}

Physical Security ensures operability and effectiveness tests of all installed security systems at Category I and II SNM facilities are conducted according to DOE directives at prescribed intervals (Table 1).

Physical Security maintains results of all the security system performance tests. If there is a problem with a component, immediate actions are taken to either repair the system or provide compensatory measures. If compensatory measures are expected to exceed 5 days, a corrective 
Table 1. Essential System Element Identification and Testing. (5 sheets)

\begin{tabular}{|c|c|c|c|c|c|c|c|}
\hline \multirow[b]{2}{*}{ Description } & \multirow[b]{2}{*}{ Each shift } & \multicolumn{6}{|c|}{ Test frequency/type/performed by } \\
\hline & & Weekly & Monthly & Quarterly & Semiannually & Annually & Requirement \\
\hline Interior microwave Grade I & $\frac{\text { Operability }}{\text { Patrol - CAS }}$ & Operability & -- & - & $\frac{\text { Effectiveness }}{\text { Sec Testing }}$ & $\begin{array}{l}\text { PM - Sec } \\
\text { Maintenance }\end{array}$ & $\begin{array}{l}\text { DOE M } 5632.1 C-1^{t} \\
\text { RL Perf. Spec }\end{array}$ \\
\hline Bal arced magnetic switch Grade I & $\begin{array}{l}\text { Operability } \\
\text { On Line } \\
\text { Patrol }\end{array}$ & $\frac{\text { Operability }}{\text { Plant }}$ & - & - & $\begin{array}{l}\text { Effectiveness } \\
\text { Sec Testing }\end{array}$ & - & $\begin{array}{l}\text { DOE M 5632.1C-1 } \\
\text { RL Perf. Spec }\end{array}$ \\
\hline Dual tech interior Grade I & $\frac{\text { Operability }}{\text { Patrol - CAS }}$ & $\frac{\text { Operability }}{\text { Plant }}$ & - & -- & $\frac{\text { Effectiveness }}{\text { Sec Testing }}$ & $\begin{array}{l}\text { PM - Sec } \\
\text { Maintenance }\end{array}$ & $\begin{array}{l}\text { DOE M } 5632.1 \mathrm{C}-\mathrm{1}^{1} \\
\text { RL Perf. Spec }\end{array}$ \\
\hline Tamper alarms & - & -- & $-\cdot$ & -- & $\begin{array}{l}\text { Effectiveness } \\
\text { Sec Maint }\end{array}$ & $\begin{array}{l}\text { PM - Sec } \\
\text { Maintenance }\end{array}$ & $\begin{array}{l}\text { DOE M 5632.1C-1' } \\
\text { RL Perf Spec }\end{array}$ \\
\hline $\begin{array}{l}\text { Interior closed-circuit television } \\
\text { motion detection }\end{array}$ & $\begin{array}{l}\text { Operability } \\
\text { Patrol - CAS }\end{array}$ & - & -- & .. & $\begin{array}{l}\text { Effectiveness } \\
\text { Sec Testing } \\
\end{array}$ & $\begin{array}{l}\text { PM - Sec } \\
\text { Maintenance } \\
\end{array}$ & $\begin{array}{l}\text { DOE M } 5632.1 \mathrm{C}-1^{1} \\
\text { RL Perf Spec }\end{array}$ \\
\hline UPS & $\begin{array}{l}\text { Operability } \\
\text { Patrol-CAS }\end{array}$ & -- &.- & -- & $\begin{array}{l}\text { Effectiveness } \\
\text { Sec Maint }\end{array}$ & $\begin{array}{l}\text { PM: Scc } \\
\text { Maintenance }\end{array}$ & $\begin{array}{l}\text { DOE M } 5632.1 \mathrm{C}-1^{\prime} \\
\text { RL Perf Spec }\end{array}$ \\
\hline Bistatic microwave & $\frac{\text { Operability }}{\text { Patrol - CAS }}$ & $\begin{array}{l}\text { Operability } \\
\text { Patrol - Walk } \\
\text { Test }\end{array}$ & $-\cdot$ & - & $\frac{\text { Effectiveness }}{\text { Sec Testing }}$ & $\begin{array}{l}\text { PM - Sec } \\
\text { Maintenance }\end{array}$ & $\begin{array}{l}\text { DOE M 5632.1C-1' } \\
\text { RL Perf Spec }\end{array}$ \\
\hline Breakwire & $\frac{\text { Operability }}{\text { Patrol - CAS }}$ & - & - & - & $\frac{\text { Efrectiveness }}{\text { Sec Testing }}$ & - & $\begin{array}{l}\text { DOE M 5632.1C-1 } \\
\text { RL Perf Spec }\end{array}$ \\
\hline E-Field & $\frac{\text { Operability }}{\text { Patrol - CAS }}$ & $\begin{array}{l}\frac{\text { Operability }}{\text { Patrol - Walk }} \\
\text { Test }\end{array}$ & - & $\begin{array}{l}\mathrm{PM}-\mathrm{Sec} \\
\text { Maintenance }\end{array}$ & $\frac{\text { Effectiveness }}{\text { Sec Testing }}$ & - & $\begin{array}{l}\text { DOE M 5632.1C-1' } \\
\text { RL Perf Spec }\end{array}$ \\
\hline Passive $\mathbb{R}$ & $\frac{\text { Operability }}{\text { Patrol - CAS }}$ & $\frac{\text { Operability }}{\text { Plant }}$ & - & $\begin{array}{l}\text { PM - Sec } \\
\text { Maintenance }\end{array}$ & $\frac{\text { Effectiveness }}{\text { Sec Testing }}$ & - & $\begin{array}{l}\text { DOE M 5632.1C-1 } \\
\text { RL Perf Spec }\end{array}$ \\
\hline Vibration detector & $\frac{\text { Operability }}{\text { Patrol - CAS }}$ & $\frac{\text { Operability }}{\text { Patrol }}$ & - & - & $\begin{array}{l}\text { Effectiveness } \\
\text { Sec Testing }\end{array}$ & $\begin{array}{l}\text { PM - Sec } \\
\text { Maintenance }\end{array}$ & $\begin{array}{l}\text { DOE M } 5632.1 C-1^{1} \\
\text { RL Perf Spec }\end{array}$ \\
\hline$X$-ray & $\frac{\text { Operability }}{\text { Patrol }}$ & -- & $\begin{array}{l}\text { Image } \\
\text { Resolution } \\
\text { Sec Testing }\end{array}$ & $\begin{array}{l}\text { PM - Sec } \\
\text { Maintenance }\end{array}$ & - & - & $\begin{array}{l}\text { DOE M 5632.1C-1 } \\
\text { RL Perf Spec }\end{array}$ \\
\hline
\end{tabular}


Table 1. Essential System Element Identification and Testing. (5 sheets)

\begin{tabular}{|c|c|c|c|c|c|c|c|}
\hline & & \multicolumn{6}{|c|}{ Test frequency/type/performed by } \\
\hline Description & Each shift & Weckly & Monthly & Quarterly & Semiannually & Annually & Requirement \\
\hline SNM personnel & - & $\frac{\text { Operability }}{\text { Plant }}$ & - & $\frac{\text { Effectiveness }}{\text { Sec Testing }}$ & $\begin{array}{l}\mathrm{PM}-\mathrm{Sec} \\
\text { Maintenance }\end{array}$ & - & $\begin{array}{l}\text { DOE M } 5632.1 \mathrm{C}-1^{1} \\
\text { RL Perf Spec }\end{array}$ \\
\hline SNM vehicle & - & $\begin{array}{l}\text { Operability } \\
\text { Plant/Sec } \\
\text { Testing }\end{array}$ & - & $\frac{\text { Effectiveness }}{\text { Sec Testing }}$ & $\begin{array}{l}\text { PM-Sec } \\
\text { Maintenance }\end{array}$ & - & $\begin{array}{l}\text { DOE M S632.1C.1 } \\
\text { RL Perf Spec }\end{array}$ \\
\hline SNM handheld & $\frac{\text { Operability }}{\text { Patrol }}$ & - & $\frac{\text { Effectiveness }}{\text { Sec Testing }}$ & - & - & - & $\begin{array}{l}\text { DOE M 5632.1C-1 } \\
\text { RL Perf Spec }\end{array}$ \\
\hline $\begin{array}{l}\text { Protected Area barriers - fences } \\
\text { and gates }\end{array}$ & - & - & - & - & - & $\begin{array}{l}\text { Internal } \\
\text { Assessments }\end{array}$ & DOE M 5632.1C-1' \\
\hline $\begin{array}{l}\text { Material Access Area barriers - } \\
\text { doors, walls, floors, and ceilings }\end{array}$ & - & - & - & - & - & $\begin{array}{l}\text { Internal } \\
\text { Assessments }\end{array}$ & DOE M 5632.1C-1 ${ }^{1}$ \\
\hline Metal - portal & $\frac{\text { Opcrability }}{\text { Patrol }}$ & $\begin{array}{l}\text { Effectiveness } \\
\text { Sec Testing }\end{array}$ & - & $\frac{\text { Effectiveness }}{\text { Sec Testing }}$ & - & $\begin{array}{l}\text { Effectiveness } \\
\text { Sec Testing }\end{array}$ & $\begin{array}{l}\text { DOE M 5632.1C-1' } \\
\text { RL Perf Spec }\end{array}$ \\
\hline Metal - handheld & $\frac{\text { Operability }}{\text { Patrol }}$ & - & $\begin{array}{l}\text { Efrectiveness } \\
\text { Sec Testing }\end{array}$ & - & - & $\rightarrow$ & $\begin{array}{l}\text { DOE M } 5632.1 \mathrm{C}-1^{1} \\
\text { RL Perf Spec }\end{array}$ \\
\hline Durcss - DES & $\frac{\text { Operability }}{\text { Patrol CAS }}$ & - & $\frac{\text { Opcrability }}{\text { Sec Testing }}$ & - & - & $\cdots$ & $\begin{array}{l}\text { DOE M 5632.1C-1' } \\
\text { DOE M 5632.7A }\end{array}$ \\
\hline $\begin{array}{l}\text { SPO II - Plutonium Finishing } \\
\text { Plant/Fast Flux Test Facility/100K }\end{array}$ & - & - & $\begin{array}{l}\text { FTO } \\
\text { Patrol }\end{array}$ & - & - & $\begin{array}{l}\text { Internal } \\
\text { Assessment } \\
\text { Performance } \\
\text { Testing }\end{array}$ & $\begin{array}{l}\text { DOE M } 5632.7 \mathrm{~A}^{2} \\
\text { DOE Order } 470.1^{3} \\
\text { CM-4-55 }\end{array}$ \\
\hline SPO III & - & - & $\begin{array}{l}\text { FTO } \\
\text { Patrol }\end{array}$ & - & - & $\begin{array}{l}\text { Internal } \\
\text { Assessment } \\
\text { Performance } \\
\text { Testing }\end{array}$ & $\begin{array}{l}\text { DOE M } 5632.7 A^{2} \\
\text { DOE Order } 470.1^{3} \\
\text { CM-4-55 }\end{array}$ \\
\hline $\begin{array}{l}\text { Personnel Security Assurance } \\
\text { Program }\end{array}$ & - & - & - & - & - & $\begin{array}{l}\text { Intemal } \\
\text { Assessment }\end{array}$ & $\begin{array}{l}\text { DOE Order } 470.1^{3} \\
\text { DOE Order } 5633.3 B^{5}\end{array}$ \\
\hline
\end{tabular}


Table 1. Essential System Element Identification and Testing. (5 sheets)

\begin{tabular}{|c|c|c|c|c|c|c|c|}
\hline \multirow[b]{2}{*}{ Description } & \multirow[b]{2}{*}{ Each shift } & \multicolumn{6}{|c|}{ Test frequency/type/performed by } \\
\hline & & Weckly & Monthly & Quarterly & Semiannually & Annually & Requirement \\
\hline $\begin{array}{l}\text { Access control to Category I and } \\
\text { Category II SNM }\end{array}$ & -- & -- & $\begin{array}{l}\text { Opcrability } \\
\text { MBA audits, } \\
\text { RAA }\end{array}$ & -- & - & $\begin{array}{l}\text { Effcctiveness } \\
\text { Internal } \\
\text { assessments } \\
\text { and MBA } \\
\text { audits, RAA }\end{array}$ & $\begin{array}{l}\text { DOE Order } 470.1^{3} \\
\text { DOE M } 5633.3 B^{3} \\
\text { Implementation } \\
\text { Guide } \\
\text { DOE MC\&A. } \\
\text { Detection Elements } \\
\text { Guide }^{7}\end{array}$ \\
\hline $\begin{array}{l}\text { Matcrial surveillance activitics for } \\
\text { Catcgory I and Category II SNM }\end{array}$ & -- & .- & $\begin{array}{l}\text { Opcrability } \\
\text { MBA audits, } \\
\text { RAA }\end{array}$ & -- & - & $\begin{array}{l}\text { Effectiveness } \\
\text { Internal } \\
\text { assessments } \\
\text { and MBA. } \\
\text { audits, RAA }\end{array}$ & $\begin{array}{l}\text { DOE Order } 470.1^{3} \\
\text { DOE M } 5633.3 B^{5} \\
\text { Implementation } \\
\text { Guide }^{6} \\
\text { DOE MC\&A } \\
\text { Detection Elements } \\
\text { Guide? }\end{array}$ \\
\hline $\begin{array}{l}\text { Tamper-indicating device control } \\
\text { and usage }\end{array}$ & -. & -- & $\begin{array}{l}\text { Operability } \\
\text { MBA audits, } \\
\text { RAA }\end{array}$ & & $\begin{array}{l}\text { Operability } \\
\text { Safeguards } \\
\text { and forms } \\
\text { administrators }\end{array}$ & $\begin{array}{l}\text { Effectiveness } \\
\text { Internal } \\
\text { assessments } \\
\text { and MBA } \\
\text { audits, RAA }\end{array}$ & $\begin{array}{l}\text { DOE Order } 470.1^{3} \\
\text { DOE M } 5633.3 \mathrm{~B}^{5} \\
\text { Implementation } \\
\text { Guide }^{\mathrm{s}} \\
\text { DOE MC\&A } \\
\text { Detection Elements } \\
\text { Guide }^{7}\end{array}$ \\
\hline Physical inventory & $\begin{array}{l}\text { Operability } \\
\text { Daily Cat I } \\
\text { NMC and } \\
\text { operations }\end{array}$ & -- & $\begin{array}{l}\text { Operability } \\
\text { NMC and } \\
\text { safeguards } \\
\text { Witness } \\
\text { MBA audits, } \\
\text { RAA }\end{array}$ & -- & .• & $\cdot$ & DOE M 5633.3Bs \\
\hline $\begin{array}{l}\text { Portal monitoring MC\&A } \\
\text { oversight }\end{array}$ & - & - & $\begin{array}{l}\frac{\text { Operability }}{\text { doc. review }} \\
\text { MBA audits, } \\
\text { RAA }\end{array}$ & -- & - & $\begin{array}{l}\text { Effectiveness } \\
\text { intemal } \\
\text { assessments } \\
\text { and MBA } \\
\text { audits, RAA }\end{array}$ & $\begin{array}{l}\text { DOE Order } 470.1^{3} \\
\text { DOE M } 5633.3 B^{3} \\
\text { Implementation Guide } \\
\text { for } 5633.3 B^{6} \\
\text { DOE MC\&A } \\
\text { Detection Elements } \\
\text { Guide }^{7}\end{array}$ \\
\hline
\end{tabular}


Table 1. Essential System Element Identification and Testing. (5 sheets)

\begin{tabular}{|c|c|c|c|c|c|c|c|}
\hline \multirow[b]{2}{*}{ Description } & \multirow[b]{2}{*}{ Each shift } & \multicolumn{6}{|c|}{ Test frequency/type/performed by } \\
\hline & & Weckiy & Monthly & Quarterly & Semiannually & Annually & Requirement \\
\hline Accounting records and inventory & -- & - & $\begin{array}{l}\text { Operability } \\
\text { MBA audits, } \\
\text { RAA }\end{array}$ & -- & -. & $\begin{array}{l}\text { Effectiveness } \\
\text { Internal } \\
\text { assessments } \\
\text { and MBA } \\
\text { audits, RAA }\end{array}$ & $\begin{array}{l}\text { DOE Order } 470.1^{3} \\
\text { DOE M } 5633.3 \mathrm{~B}^{5} \\
\text { Implementation Guide } \\
\text { for } 5633.3 \mathrm{~B}^{6} \\
\text { DOE MC\&A } \\
\text { Detection Elements } \\
\text { Guide }^{7}\end{array}$ \\
\hline $\begin{array}{l}\text { Inventory confirmation/verification } \\
\text { measurements } \\
\end{array}$ & -- & - & $\begin{array}{l}\frac{\text { Operability }}{\text { doc. review }} \\
\text { MBA audits, } \\
\text { RAA }\end{array}$ & -. & -- & $\begin{array}{l}\text { Effectiveness } \\
\text { Internal } \\
\text { assessments } \\
\text { and MBA } \\
\text { audits, RAA }\end{array}$ & $\begin{array}{l}\text { DOE Order } 470.1^{3} \\
\text { DOE M } 5633.3 B^{3} \\
\text { Implementation Guide } \\
\text { for } 5633.3 B^{6} \\
\text { DOE MC\&A } \\
\text { Detection Elements } \\
\text { Guide }^{7}\end{array}$ \\
\hline Inventory difference control limits & -- & -- & $\begin{array}{l}\frac{\text { Operability }}{\text { doc. review }} \\
\text { MBA audits, } \\
\text { RAA }\end{array}$ & -- & -- & $\begin{array}{l}\text { Effcctiveness } \\
\text { Intemal } \\
\text { assessments } \\
\text { and MBA } \\
\text { audits, RAA }\end{array}$ & $\begin{array}{l}\text { DOE Order } 470.1^{3} \\
\text { DOE M } 5633.3 \mathrm{~B}^{3} \\
\text { Implementation Guide } \\
\text { for } 5633.3 \mathrm{~B}^{6} \\
\text { DOE MC\&A } \\
\text { Detection Elements } \\
\text { Guide }^{7}\end{array}$ \\
\hline $\begin{array}{l}\text { Other MC\&A performance } \\
\text { indicators specified }\end{array}$ & - & - & $\begin{array}{l}\text { Operability } \\
\text { MBA audits, } \\
\text { RAA }\end{array}$ & -- & -- & $\begin{array}{l}\text { Effectiveness } \\
\text { Internal } \\
\text { assessments } \\
\text { and MBA } \\
\text { audits, RAA }\end{array}$ & $\begin{array}{l}\text { DOE Order } 470.1^{3} \\
\text { DOE M } 5633.3 \mathrm{~B}^{3} \\
\text { implementation Guide } \\
\text { for } 5633.3 \mathrm{~B}^{6} \\
\text { DOE MC\&A } \\
\text { Detection Elements } \\
\text { Guide }^{7}\end{array}$ \\
\hline $\begin{array}{l}\text { Site-specific performance } \\
\text { evaluations }\end{array}$ & -- & - & $\begin{array}{l}\text { Operability } \\
\text { MBA audits, } \\
\text { RAA }\end{array}$ & - & -- & $\begin{array}{l}\text { Effectiveness } \\
\text { Internal } \\
\text { assessments } \\
\text { and MBA } \\
\text { audits, RAA }\end{array}$ & $\begin{array}{l}\text { DOE Order } 470.1^{3} \\
\text { DOEM } 5633.3 B^{5} \\
\text { Implementation Guide } \\
\text { for } 5633.3 B^{6} \\
\text { DOE MC\&A } \\
\text { Detection Elements } \\
\text { Guide }^{7}\end{array}$ \\
\hline
\end{tabular}


Table 1. Essential System Element Identification and Testing. ( 5 sheets)

\begin{tabular}{|c|c|c|c|c|c|c|c|}
\hline & & \multicolumn{5}{|c|}{ Test frequency/type/performed by } \\
\hline Description & Each shif & Weekly & Monthly & Quarterly & Semiannually & Annually & Requirement \\
\hline
\end{tabular}

'DOE, 1994b, Manual for Protection and Control of Safeguards and Security Interests, DOE M 5632.1C-1, U.S. Department of Energy, Washington, D.C.

${ }^{2} \mathrm{DOE}, 1995 \mathrm{c}$, Protective Force Program, DOE M 5632.7A, U.S. Department of Energy, Washington, D.C.

${ }^{3}$ DOE, 1995a, Safeguards and Security Program, DOE Order 470.1, U.S. Department of Energy, Washington, D.C.

${ }^{4} \mathrm{HNF}$-CM-4-S5, Patrol Policies, Procedures, and Training, Fluor Danicl Hanford, Inc., Richland, Washington.

${ }^{3} \mathrm{DOE}, 1994 \mathrm{c}$, Control and Accountability of Nuclear Materials, DOE Order 5633.3B, U.S. Department of Energy, Washington, D.C.

${ }^{\circ} \mathrm{DOE}, 1995 \mathrm{~d}$, Guide for Implementation of DOE 5633.3B, "Control and Accountability of Nuclear Materials," U.S. Department of Energy, Office of Security. Affairs, Office of Safeguards and Security, Washington, D.C.

${ }^{7} \mathrm{DOE}, 1994 \mathrm{~d}$, Guide to the Evaluation of Selected Material Control and Accountability (MC\&A) Detection Elements, U.S. Department of Energy, Office of Security Affairs, Office of Safeguards and Security, Washington, D.C.

CAS $=$ Central Alarm Station

Cat I, II = category of nuclear material

DES $=$ Digital Encryption System

FPS $=$ Fence Protection System

FTO $=$ Ficld Training Oflicer

$\mathbb{R}=$ infrared

$\mathrm{MBA}=$ Material Balance Area

$M C \& A=$ material control and accountability

o $\quad \mathrm{NMC}=$ nuclear material custodian

$\mathrm{PM}=$ preventive maintenance

RAA $=$ Risk Analysis \& Assessments

SNM = special nuclear material

SPO $=$ Security Police Officer

UPS $=$ uninterruptible power supply 
action plan will be developed and forwarded to Risk Analysis \& Assessments (RAA) within 10 working days after completion of the test.

\subsubsection{Hanford Patrol Training Academy}

The Hanford Patrol Training Academy conducts field training exercises frequently. These exercises are designed to evaluate the Protective Force on its job responsibilities. Results of the exercises are used as a basis for training development and to fulfill the requirements for assessment and alarm response to Category I and II SNM facilities.

The Patrol Training Academy provides training for exercise controllers and evaluators, Field Training Officer support for LSPTs, and other comprehensive performance tests.

\subsubsection{Hanford Patrol}

The Hanford Patrol conducts operability tests at the prescribed intervals of the Perimeter Intrusion Detection and Assessment System, interior sensors, X-ray unit(s), SNM detectors, and metal detector(s). Plant personnel also conduct operability tests of installed interior security systems at prescribed intervals. These results are maintained by Physical Security.

\subsubsection{Safeguards}

Safeguards ensures any anomaly, that is any deviation from normal or acceptable practices relating to the control and accountability of nuclear material in the course of normal operations, is identified and resolved promptly (reference, HNF-PRO-610, "Safeguards Anomaly Report", dated October 1, 1996). Identified anomalies are included in the BWP Corrective Action Management Program and tracked by Safeguards until complete. All corrective actions are validated by Risk Analysis and Assessments prior to being closed.

As an integral part of the Performance Assurance Program, Safeguards, in conjunction with Risk Analysis and Assessments, validates the functional requirements and system effectiveness of MC\&A elements as part of the total safeguards and security system. This validation is completed as part of the BWP Internal Assessment and Performance Testing programs. Specific essential elements have been identified and will be tested as indicated in Table 1.

\subsubsection{Emergency Preparedness}

Emergency Preparedness conducts many functional drills, limited exercises, tabletop exercises, field exercises, and area evacuations each year in accordance with DOE directives. Physical Security, RAA, and Hanford Patrol are members of the Scenario Development Group for major Site exercises. Any SAS needs are taken into consideration and incorporated into the scenario planning. 


\subsubsection{Risk Analysis \& Assessments}

The BWP RAA organization plans, schedules, and coordinates annual major comprehensive performance tests of the essential system elements of each Category I and Category II SNM facility with credible rollup to Category I for FDH SAS systems. These tests will ascertain the effectiveness in providing countermeasures to address the design basis threat as outlined in the DOE Design Basis Threat Policy for Department of Energy Programs and Facilities (DOE 1994a). A formal test report is prepared, along with appropriate corrective actions, and provided to FDH for RI SAS. Corrective actions are tracked internally by RAA.

The BWP RAA organization also plans, schedules, and performs annual internal audits of Category I and II SNM Material Balance Areas (MBA). The essential system elements evaluated include nuclear material custodian selection and approval, nuclear material transfer controls and documentation, physical inventories, measurement control, access control, material surveillance and containment, tamper-indicating devices, and forms control. A summary report is prepared and distributed, along with appropriate findings and/or suggestions for FDH approval and submittal to the subcontractor President, Plant Manager, and nuclear material custodian for action. Corrective actions to findings are tracked within the Hanford Action Tracking System. Followup to suggestions is completed during the subsequent MBA audit. The effectiveness of the MC\&A program is also evaluated during annual internal assessments, RL SAS surveys, and U.S. Department of Energy-Headquarters evaluations.

The BWP RAA organization is responsible to $\mathrm{FDH}$ for the coordination of material control and accountability performance tests to ensure the effectiveness of essential elements. These tests are completed during MBA audits, internal assessments, vulnerability analyses, systems security tests, and observations of MBA operations by Safeguards personnel. The performance tests evaluate the effectiveness of access control, material surveillance, the tamperindicating devices program, portal monitoring, accounting record system, inventory confirmation, and verification measurements for Category I and II SNM. Results of performance tests that require corrective actions are tracked internally by RAA on the SAS Punchlist until completion.

\subsection{PROGRAM REQUIREMENTS}

This plan is the formal PHMC program plan for the SAS Performance Assurance Program. B\&W Protec, Inc., ensures updates to the plan are made as required, but as a minimum the plan is reviewed annually. Each year, BWP provides the completed plan to FDH for approval and FDH submission to RL SAS.

Essential system elements will be identified for each program element according to DOE Order 470.1 to include the following: personnel security, protection program operations, MC\&A, and information security. These elements will be tested by appropriate groups to verify their continued functionability, operability, effectiveness, and/or performance. Usually, verification will be accomplished during annual assessments of each particular function. 
However, in some cases, when adequate test data-are not current or available, other LSPTs may be required, e.g., MC\&A, Protective Forces, vulnerability assessment validation, and security system performance testing.

At least annually, BWP will conduct a comprehensive performance test encompassing essential system elements of each Category I or II SNM facility. A quantitative analysis of performance test results will be conducted, when appropriate, using a Likert Scale (Likert 1932) evaluation tool (see Appendix D) and then converting the results of the evaluation to a numerical equivalence on a value matrix.

Physical Security will conduct operability tests to provide a simple measure of integrity of security systems. The testing frequency of essential system elements will be according to HNFIP-0921, Operability Testing (BWP 1997a). The tests will confirm, without any indication of effectiveness, that the system is operating.

Effectiveness tests provide comprehensive assurance of integrity. Effectiveness testing of essential system elements will confirm the satisfactory performance of the required functions over the expected range of use. The testing will be conducted in accordance with HNF-IP-0920, Effectiveness Test Procedure (BWP 1997b).

At least annually, or immediately after an inoperative (down/fail) state, and/or immediately after repair of each critical system element, the system will be tested by means of an effectiveness test.

Test documentation will be retained in accordance with DOE Order 1324.5B, Records Management Program (DOE 1995b). Record-keeping systems will provide an audit trail showing the relationship among all test data and test documentation.

\subsection{ESSENTIAL SYSTEM ELEMENTS}

Essential system element identification and testing requirements are shown in Table 1.

\subsection{TEST DOCUMENTATION REQUIREMENTS}

\subsection{REQUIRED TEST PLANS}

Each activity conducting a performance test is responsible for development of specific test plans (for example, Physical Security when performing an effectiveness test, Hanford Patrol Training Academy when conducting LSPTs of the Protective Forces, and RAA when conducting annual comprehensive performance tests). Test plans for annual comprehensive performance tests will follow the outline in Appendix A. An approved performance test safety plan must also be completed and approved before any test. The scope of the test will determine the depth of the safety plan requirements. Performance test safety plan coordination and approval will follow the 
guidelines in Appendix A. Test plans for other types of performance tests may be individually tailored but should include the following as a minimum:

- Test objective

- Test methodology

- Pass/fail criteria

- Data analysis

- Test controls

- Resources

- Test coordination and approval process

- Operational impacts

- Compensatory measures

- References.

\subsection{REQUIRED TEST REPORTS}

Test results for annual comprehensive performance tests will be formally documented in a test report. Each test report will follow the outline in Appendix B and be maintained on file within BWP, RAA files. Results of tests conducted by activities other than RAA will be maintained with that organization unless the results show major deficiencies, in which case, the results will be forwarded to RAA. Results of emergency preparedness exercises dealing with SAS interests will be forwarded to RAA. Reports will show any major deficiencies, corrective actions, persons responsible for correcting the deficiencies, and compensatory measures taken. All performance test reports will be reviewed for classification by an Authorized Derivative Classifier.

\subsection{TEST APPROVALS}

Annual comprehensive test and safety plans for Category I and II facilities will be approved at least 10 days before the test by the following:

- Lead, RAA Performance Testing

- Appropriate vulnerability analysis lead

- Manager, RAA

- Authorized Derivative Classifier

- Area Security Representative

- Manager, Site Security Operations

- Chief, Hanford Patrol

- Senior Controller

- Safety Administrator, BWP

- Plant Safety Officer

- Manager, Plant Operations

- Manager, Safeguards and Planning

- Director, BWP

- Director, FDH, Infrastructure \& Security

- Director, RL SAS. 


\subsection{CORRECTIVE ACTION REQUIREMENTS}

\subsection{DEFICIENCIES IENTIFIED DURING PERFORMANCE TESTING}

Deficiencies identified during comprehensive performance tests and LSPTs conducted at Category I or II SNM facilities shall be formally documented and corrective actions tracked by BWP until resolved. A formal corrective action plan shall be provided to BWP within 20 working days after receipt of the report. Responsibility for the ultimate resolution of identified deficiencies shall reside with the appropriate FDH and subcontractor organizations. Deficiencies identified during security systems performance testing and emergency preparedness drills that do not result in a systems failure shall be documented and tracked by the responsible activity until corrected. Refer to Section 8.2 for deficiencies resulting in a systems failure.

\subsection{TEST FAILURES}

A test failure of an essential system component identified in Table I will require immediate implementation of compensatory measures. A failure is a condition in which a system or procedure is not performing or being followed effectively. Failures will be reported to the respective functional manager. If compensatory measures are expected to exceed 5 days, a corrective action plan will be developed and forwarded to RAA within 10 working days after completion of the test.

Corrective action plans will show the deficiency, what corrective actions are being taken/planned, compensatory measures, persons responsible for correcting the deficiencies, completion date, and any milestones. The RAA organization will track corrective actions to completion.

\subsection{RESOURCES}

\subsection{PHYSICAL SECURITY AND SECURITY ENGINEERING AND MAINTENANCE}

Physical Security and Security Engineering and Maintenance will maintain a sufficient number of trained personnel to conduct systems performance testing and maintenance of essential protection elements. 


\subsection{RISK ANALYSIS \& ASSESSMENTS}

The RAA organization will ensure trained personnel are assigned to administer the Performance Assurance Program, track corrective actions to completion, and ensure required annual performance testing is completed to sufficiently validate new and existing vulnerability analyses of Category I and II SNM facilities.

\subsection{HANFORD PATROL}

Hanford Patrol will ensure sufficient resources are available to complete the required number of LSPTs, field training exercises and annual comprehensive performance tests as outlined in WHC-SP-1163, Safeguards and Security Fiscal Year Program Plan (WHC 1996) and this document.

\subsection{REFERENCES}

BWP, 1997a, Operability Testing, HNF-IP-0921, July 29, B\&W Protec, Inc., Richland, Washington.

BWP, 1997b, Effectiveness Test Procedwe, HNF-IP-0920, May 1, B\&W Protec, Inc., Richland, Washington.

DOE, 1994a, Design Basis Threat Policy for Department of Energy Programs and Facilities, RL-HQ-00649, U.S. Department of Energy, Washington, D.C.

DOE, 1994b, Mamual for Protection and Control of Safeguards and Security Interests, DOE M 5632.1C-1, U.S. Department of Energy, Washington, D.C.

DOE, 1994c, Commol and Accommability of Nuclear Materials, DOE Order 5633.3B, U.S. Department of Energy, Washington, D.C.

DOE, 1994d, Guide to the Evaluation of Selected Material Conirol and Accountability (MC\&A) Detection Elements, U.S. Department of Energy, Office of Security Affairs, Office of Safeguards and Security, Washington, D.C.

DOE, 1995a, Safeguards and Security Program, DOE Order 470.1, U.S. Department of Energy, Washington, D.C.

DOE, 1995b, Records Management Program, DOE Order 1324.5B, U.S. Department of Energy, Washington, D.C.

DOE, 1995c, Protective Force Program, DOE Order 5632.7A, U.S. Department of Energy, Washington, D.C. 
DOE, 1995d, Guide for Implemeitation of DOE 5633.3B, "Control and Accountability of Nuclear Materials, "U.S. Department of Energy, Office of Security Affairs, Office of Safeguards and Security, Washington, D.C.

HNF-CM-4-55, Patrol Policies, Procedures, and Training, Fluor Daniel Hanford, Inc., Richland, Washington.

HNF-PRO-610, Safeguards Anomaly Report, Fluor Daniel Hanford, Inc., Richland, Washington.

Likert, R., 1932, "A Technology for the Measurement of Attitudes," Archives of Psychology, 140.

RL, 1992, Hanford Site Safeguards and Security Performance Specifications: Field Testing Criteria for Installed Intrusion Detection Sensors, June, U.S. Department of Energy, Richland Operations Office, Rịchland, Washington.

WHC, 1996, Safeguards and Security Fiscal Year Program Plan, WHC-SP-1163, Westinghouse Hanford Company, Richland, Washington.

\subsection{BIBLIOGRAPHY}

DDOE, 1996, DOE Performaince Assurance Program Supplement, November, U.S. Department of Energy, Washington, D.C.

RL, 1993, SAS-92-02, Hanford Site Safeguards and Security Performance Specifications: Field Testing Criteria for EntryiExit Control Screening Devices, U.S. Department of Energy, Richland Operations Office, Richland, Washington. 
HNF-EP-0927

\section{APPENDIX A}

EXAMPLE - PERFORMANCE TEST PLAN

A-1 
HNF-EP-0927

This page intentionally left blank.

A-2 
HNF-EP-0927

\section{APPENDIX A \\ EXAMPLE - PERFORMANCE TEST PLAN}

1. Test Objectives. The objective(s) of this performance test plan will be:

2. Scenario Description
A. Threat scenario
B. Systems to be evaluated
C. Boundaries.

\section{Test Methodology and Evaluation Criteria}

A. Conduct of test. Show how the test will be evaluated, by whom, and who has control over actual conduct of the exercise.

B. Planning. The Risk Analysis \& Assessments (RAA) organization will take the following steps when it develops the performance test.

(1) Research, develop, and write a complete formal test plan.

a. Coordinate with Protective Force senior controller operations to determine specific assignments and responsibilities.

b. Coordinate with the B\&W Protec, Inc. (BWP) Safety Administrator and appropriate facility safety coordinators to ensure all safety considerations have been identified and properly integrated into planning to prevent undue risk to personnel.

c. Ensure logistical requirements have been identified.

d. Ensure the U.S. Department of Energy, Richland Operations Office (RL) Safeguards and Security (SAS) personnel are involved in the development of the plan.

e. Ensure Facility Operations is fully apprised of test schedule and scenario.

(2) Ensure authorized derivative classification review before routing.

(3) Route test and safety plans for review and approval by BWP; Fluor Daniel Hanford, Inc. (FDH); and RL.

(4) Place original signature copy of the approved test plan in a master file and prepare a working file for field use.

C. Execution. List each step in the execution of the test. 
D. Equipment effectiveness (applicable to systems security. testing and maintenance).

E. Number of tests to be conducted.

F. Pass/fail criteria. Identify the specific pass/fail criteria for each objective being tested.

G. Data analysis. Example:

\begin{tabular}{|c|c|c|c|c|c|c|c|c|c|c|c|}
\hline COMMUNICATIONS & 0 & 1 & 2 & 3 & 4 & 5 & 6 & 7 & 8 & 9 & 10 \\
\hline $\begin{array}{l}\text { 1. How effective is the communications equipment? } \\
\text { 2. Extent of effectiveness of communications for clarity, conciseness, and accuracy. } \\
\text { 3. How timely were communications? } \\
\text { 4. How timely.were requests for assistance? }\end{array}$ & & & & & & & & & & & \\
\hline
\end{tabular}

4. Test controls. Indicate what test controls will be in place to ensure the integrity of the test and ensure safety and security risks are minimized.

A. The test coordinator has primary responsibility for test coordination and will ensure the performance test and safety plans have been approved before the test. The test coordinator will accompany the senior controller during the test.

B. The senior controller will have primary responsibility and authority for the conduct of the test and will ensure the security and safety of all government property and personnel is not degraded. Each test participant has the responsibility to observe all safety and security rules and immediately call a "HALT" to the test if a safety or security violation is observed. Each participant will also observe correct communications discipline and start and end all communications with "THIS IS A TEST MESSAGE."

C. There will be a controller with each Engagement Simulation System (ESS) weapon. The controller will ensure weapons are modified according to U.S. Department of Energy directives and that proper safety practices are followed during the test.

D. General controller responsibilities are outlined in Appendix C. Specific responsibilities relative to the scenario will be outlined in this section.

E. Plant Operations will provide the following:

(1) Plant Manager approval

(2) Plant briefing on test rules/safety

(3) One individual to act as insider

(4) One health physics technician to support testing

(5) Safety officer to approve and support testing. 
F. Hanford Patrol will provide the following:

(1) Trained, qualified controllers for all Protective Force units

(2) Safety briefings to all Protective Force personnel before commencement of performance test

(3) Operations representative to assist in development of the test

(4) Senior controller

(5) Patrol safety representative.

G. The BWP, RAA organization will provide the following:

(1) Test coordinator

(2) Trained, qualified evaluators

(3) Management briefings

(4) Data analysis of performance test results

(5) Performance test report.

H. Physical Security will provide assistance as necessary.

I. Tests will be conducted during the most advantageous hours to the adversary as dictated by the plant vulnerability analysis.

J. Evaluators and controllers will be in positions to observe each member of the Protective Force and adversaries (if used) and any support element. Evaluators and controllers will observe/evaluate and provide test guidance, but will not direct the actions of the test players except to prevent a safety or security violation.

K. If ESS equipment is used, there will be separate safety briefings and installation safety checks. Requirements listed in DOE G 5632.7A-1 (DOE 1995) will apply if ESS equipment is used.

L. The BWP Safety Administrator will oversee test safety.

5. Resources. The following resources will be provided and actions taken as necessary:

A. RL SAS will be invited to attend every performance test.

B. Hanford Patrol

(1) Senior controller

(2) One Operations representative

(3) Sufficient number of controllers depending on number of Protective Force responders

(4) Shadow force, as necessary 
(5) Sufficient number of adversaries, as necessary

(6) Necessary training for controllers and evaluators

(7) Required ESS equipment, as necessary

(8) Safety representative.

C. RAA

(1) Test coordinator

(2) Sufficient number of trained evaluators

(3) Sufficient number of portable radios

(4) Necessary safety equipment (vests, flashlights, etc.) for evaluators and observers

(5) Management briefings

(6) Evaluator and observer briefings

(7) Approved performance test plan

(8) Approved performance test safety plan.

(9) Final performance test report

(10) Monitoring of corrective actions to major deficiencies identified

(11) Data analysis of performance test results.

D. Plant Operations

(1) One trusted agent for test planning

(2) One individual to act as insider

(3) Health physics technician

(4) Briefing room

(5) Safety Officer.

E. BWP Safety

(1) Safety Administrator.

6. Operational impact(s). Describe any operational impacts that may result from the test.
A. Operations
B. Security
C. Overtime
D. Funding.

7. Compensatory measures. Identify measures to be implemented in case of test failure.

8. Test coordination and approval process. The following coordination and approval process will be followed for all limited-scope performance tests and comprehensive performance tests. 
HNF-EP-0927

A. The Performance Test Plan shall be reviewed and approved by the following:
(1) Lead, RAA Performance Testing
(2) Appropriate vulnerability analysis lead
(3) Manager, RAA
(4) Authorized Derivative Classifier
(5) Area Security Representative
(6) Manager, Site Security Operations
(7) Chief, Hanford Patrol
(8) Senior Controller
(9) Safety Administrator, BWP
(10) Plant Safety Officer
(11) Manager, Plant Operations
(12) Manager, Safeguards and Planning
(13) Director, BWP
(14) Director, FDH, Infrastructure \& Security
(15) Director, RL SAS.

B. The Performance Test Safety Plan shall be reviewed and approved by:

Same as A. above.

C. The Performance Test Report shall be reviewed and approved by:

Same as A. above.

9. References. List pertinent requirement basis documents, standards, procedures, and reports.

\section{A1.0 REFERENCES}

DOE, 1995, Guide for Use of Protective Force Engagement Simulation Systems, DOE G 5632.7A-1, April 10, U.S. Department of Energy, Washington, D.C. 
HNF-EP-0927

This page intentionally left blank.

A-8 
HNF-EP-0927

APPENDIX B

PERFORMANCE TEST REPORT OUTLINE

B-I 
HNF-EP-0927

This page intentionally left blank. 


\section{APPENDIX B \\ PERFORMANCE TEST REPORT OUTLINE}

1. Objectives. Restate the performance test objectives listed in the performance test plan.

2. Test data. Include all recorded test data in this section.

3. Data analysis. Document the analysis of the test data using models, equations, or methodology presented in the performance test plan.

4. Test results and recommendations. This section will contain the following:

A. A statement of success (pass)/failure (fail) according to evaluation criteria contained in the test plan

B. Unusual observations: related to the area tested, but not otherwise addressed in the associated plan

C. Recommendations: actions to resolve any variations from expected test results.

5. Corrective actions. List and discuss corrective actions recommended for safeguards and security measures failing to meet requirements. Identify persons, organizations, or groups responsible for corrective actions.

A. The responsible persons, organizations, and groups will implement immediate corrective actions. Immediate corrective actions may take the form of compensatory measures until long-range resolutions are implemented.

B. Long-range corrective actions. Long-range corrective actions will be in the form of an approved corrective action plan with milestones, milestone completion dates, and the organizations or individual responsible for completing each milestone.

6. References. List the test plan and other pertinent references.

NOTE: When the performance of essential protection elements is assessed by evaluation of management oversight activities such as audits and surveys, summaries of these activities may be substituted for test reports as documentation for performance assurance activities. When appropriate, the same information contained in a test report should be included in these summaries. 


\section{HNF-EP-0927}

This page intentionally left blank.

B-4 
HNF-EP-0927

\section{APPENDIX C}

COMMAND AND CONTROL 


\section{HNF-EP-0927}

This page intentionally left blank.

C-2 


\section{APPENDIX C COMMAND AND CONTROL}

1. Every test and related activity must be regulated by controllers under the supervision of a senior controller who is responsible for overall control of the test. The senior controller must be supported by a safety controller, an Engagement Simulation System (ESS) controller, a Shadow Force controller, event controllers, and special controllers. These individuals must be adequately trained to fulfill their responsibilities to ensure activities are accomplished safely. Specific responsibilities and training requirements for these positions are discussed below.

2. Test coordinator. The Manager, Risk Analysis \& Assessments (RAA) or designated representative, is the Test Coordinator. The test coordinator is charged with responsibility for test pre-planning activities, post-test briefings and reports, and followup for any lessons learned. The test coordinator:

a. Coordinates with the Senior Controller to ensure the tasks listed in 3 below have been completed before the test

b. Ensures all appropriate safety and safeguards and security measures are in place before the start of the test

c. Ensures all required security and safety briefings are completed prior to the test.

3. Senior controller. The Chief, Hanford Patrol or designated representative is the senior controller. The senior controller is charged with overall responsibility for conduct of the test, any Patrol logistical coordination, and works directly with the test coordinator on all preplanning and post-test activities. The senior controller is responsible for coordinating, establishing, and supervising the test controller staff; identifying the number of personnel required to control the test; ensuring that appropriate controller training is conducted; and developing and implementing the concept of operation for the test coordinator. The senior controller has the following responsibilities:

a. Ensures a sufficient number of technically qualified controllers are available to support each test event and have attended onsite pre-test training and scenario orientation

b. Ensures all participant groups, as determined by the Test Coordinator, are thoroughly briefed on the test scenarios, rules of engagement, safety concerns, and emergency procedures

c. Signals the beginning and end of the test and guides and supervises all controllers and evaluators

d. Has final authority for test halts due to potential safety or safeguards and security problems 
e. Ensures test debriefings are conducted and documented:

(1) Controllers understand their responsibilities in support of test documentation.

(2) Controller debriefings are coordinated and documented.

4. Controller. The controller staff must be organized in a way that facilitates the control of all affected locations and the control and coordination of all events to be initiated during the test. The first responsibility of the controller is to ensure safety during test activity. This includes ensuring all participants follow the safety procedures and rules of engagement. If hazardous situations occur during the test, controllers must be prepared to take prompt action to prevent accidents or unsafe conditions. If these situations develop, controllers must take action in accordance with established safety procedures.

Personnel assigned as controllers are responsible for enforcing or implementing the following general requirements during tests.

a. Conduct safety checks and inspections of all personnel under their control for live rounds, prohibited articles, and general safety, and report results to the senior controller before the beginning of the test.

b. If it is an ESS test, ensure no live firearms or ammunition of any type are allowed within the test area, except those under the direct supervision of a Shadow Force controller.

c. Ensure test participants wear appropriate safety equipment.

d. Ensure personnel under their control comply with the test plan, including the rules of engagement and safety regulations.

e. Ensure personnel comply with ESS weapon handling and manipulation procedures.

f. Stop specific activity or the entire test if unsafe conditions or acts are observed.

g. Ensure the accountability of personnel and equipment at the end of the test, and report the results to the Senior Controller.

h. Ensure ESS equipment is functioning properly.

i. Ensure ESS equipment is issued and then returned promptly upon completion of the test.

5. Safety controller. The safety controller is responsible for assessing the test plan, conducting walk-downs of the test area, and conducting safety briefings that specify the rules of engagement, medical response, munitions and weapons safety, and vehicle and personnel safety. The safety controller reports to the senior controller and should remain in contact with the senior controller at all times during the test. In addition, the safety controller:

a. Assists the Senior Controller in the development and conduct of pre-test controller training 
b. Ensures adequate safety walk-downs are conducted of the area before the test

c. Ensures emergency medical and fire protection services will be present/are on call for the duration of the test.

6. ESS controller. In tests involving the use of ESS equipment, the individual assigned as ESS Controller is responsible for ensuring the following actions are completed.
a. Issue and account for all ESS firearms and support equipment.
b. Inspect all blank ammunition for the test before issue.
c. Test the ESS equipment for operability before test commencement.
d. Collect all test firearms, ESS equipment, explosive simulations, and blank ammunition at the conclusion of the test.

7. Shadow Force controller. A Shadow Force controller, who is a Protective Force supervisor with the experience necessary to ensure that the Shadow Force responds as directed to any security incident that may occur during a test, is required to ensure plant security is maintained. The Shadow Force controller is responsible for ensuring the following.

a. Voice communications are established and maintained with the senior controller throughout the test.

b. All live weapons are maintained under supervision, and Shadow Force personnel do not come in contact with test participants using ESS equipment.

c. Shadow Force personnel:

(1) Know the test area and emergency response procedures

(2) Remain under direct supervision and control during the test

(3) Are released, after coordinating with the senior controller, in case of actual alarm or other security incident, in accordance with the test plan.

8. Event controllers. Event controllers report to the senior controller and are responsible for executing control over specific categories of test activity, including one or more test events. Event controllers are responsible for ensuring nonparticipating plant personnel in the test area are aware that a test is to be conducted and not to interfere with the flow of the test. Event controllers must ensure that all test participants under their control:
a. Are aware of procedures for halting a test for safety reasons or an actual emergency
b. Are not in possession of any live weapons or ammunition 
c. Have been provided with instructions on the hazards of light anti-tank weapon simulators and any personnel using the light anti-tank weapon simulator have received comprehensive instruction on its usage before test initiation

d. All test participants are fully trained and provided with specific instruction on the hazards of special weapons, explosives, and simulators before the test

e. Have operable communications equipment

f. Are fully trained and qualified if programmed to deploy hand-thrown pyrotechnics, special weapon simulators, flash-sound diversionary devices, and/or chemical agents

g. Are instructed that if flash-sound diversionary devices are used, they are not to be thrown within $15 \mathrm{~m}(50 \mathrm{ft})$ of personnel in open areas or into occupied areas or rooms

h. All explosive simulators are returned to their point of issue at the conclusion of the test activity.

9. Special controllers. In those tests involving special weapons (e.g., light anti-tank weapon rocket simulators), explosives, pyrotechnics, etc., the test plan must specifically designate controllers for the special activity. Special controllers are responsible for ensuring that the following safety requirements are implemented, as applicable to their assigned area of involvement.

10. Evaluators. The Manager, RAA, will designate appropriate evaluators. Evaluators should have basic knowledge and experience in Protective Force operations and the general layout of the test area, basic knowledge of physical security systems, and general experience in evaluation techniques. Evaluators will be responsible for objectively evaluating actions associated with preestablished test objectives and providing a quantitative rating using the criteria and evaluation tools established in the basic plan. Additionally, evaluators will be able to detect any safety violations and act accordingly to correct the situations. Evaluators will be responsible to the senior controller.

11. Controller/evaluator training. The command and control system depends on a contingent of personnel selected and specifically trained to control force-on-force tests. Besides being trained to oversee tests, controllers and evaluators must receive training commensurate with the scope, complexity, and special nature of the activity. Hanford Patrol designates and approves all controllers and evaluators. Based on the complexity of the activity, specific controllers may be required for the Shadow Force, ESS equipment issue and accountability, occupational safety and health, and special or high-risk activities.

a. Training. All personnel assigned test controller/evaluator duties must receive documented training that includes the following topics: 
(1) Controllers/evaluator
(a) Purpose
(b) Responsibilities
(c) Duties.

(2) General knowledge
(a) ESS equipment/pyrotechnics
(b) Safety
- Firearms
- Vehicle use
- Participants
- Environment, safety, and health
- Medical
(c) Individual and team tactics
(d) Communications
(e) Application of force
(f) Command and control.

(3) Test plans
(a) Schedule
(b) Scenarios/scenario variables
(c) Protective force actions
(d) Opposition force actions
(e) Controller/evaluator actions
(f) Role player actions
(g) Suspension/resumption/termination
(h) Rules of engagement
(i) Administration
(j) Security
(k) Chain of command.

b. Scenario-specific training. Besides the training discussed above, controllers and evaluators should receive specific briefings before each test. These briefings should include the following as a minimum:

(1) The tasks and responsibilities before test initiation.

(2) Rules of engagement. Brief each controller and evaluator on the procedures for test freeze, administrative hold, rules of engagement for participants, vehicle safety, vehicle kills, explosives, weapons and ammunition, ESS equipment and capabilities, general safety, actual emergencies, and security incidents. 
(3) Documentation. Describe and demonstrate the desired method for recording information about the events that happen during the test. Describe required reports and discuss classification issues.

(4) Simulation/artificialities. Describe planned simulations and artificialities, how they will affect the test, when they will be injected, and the procedures for formulating and introducing other simulations/artificialities, as needed, after test initiation.

(5) Transportation arrangements. Describe how controllers, test participants, evaluators, data collectors, and visitors are transported to the test location.

(6) After-action meeting. Describe the purpose, location, and what information should be brought to the after-action meeting.

(7) Equipment. Describe the location for the issue and turn in of equipment, accountability measures, and detailed instructions on the equipment required for each controller and evaluator during the test.

(8) Radio usage and call signs. Provide a detailed description and demonstration of the radios the controllers and evaluators will be operating during the test. Explain the importance of operating only on the channel and frequency specified in the communications plan.

(9) Operation of ESS equipment. As appropriate, provide a detailed briefing and demonstration on each piece of ESS equipment to be used in the test and explain how and where it will be used.

(10) Route familiarization and test site. Provide each controller and evaluator with a map depicting the route to the test site and walk down the test site with all controllers and evaluators as necessary.

(11) Emergency procedures. Review and provide all controllers and evaluators with a copy of test emergency procedures.

(12) Information protection. Review the guidelines for information control and established policies and procedures for the protection of test-related classified information and unclassified controlled nuclear information.

(13) Controller and evaluator identification. Describe how controllers and evaluators will be identified and demonstrate the proper method of donning and wearing of any apparel to be used for identification purposes.

(14) Controller and evaluator meetings. Provide directions to and schedule time for any meetings. 


\section{HNF-EP-0927}

12. Trusted agents. While preparing for and conducting a test, it may be necessary to provide sensitive information to selected nonparticipants and participants regarding the occurrence and/or timing of events to accomplish necessary coordination. Such individuals may be designated as trusted agents. 


\section{HNNF-EP-0927}

This page intentionally left blank. 
HNF-EP-0927

APPENDIX D

DATA COLLECTION SHEET

D-1 
HNF-EP-0927

This page intentionally left blank. 


\section{APPENDIX D \\ DATA COLLECIION SHEET}

\section{Instructions:}

1. For each item, rate the effectiveness using the scale provided $(0-10)$, with " 0 " representing the least effective and " 10 " indicating most effective.

2. Include any qualifying remarks in the spaces provided within each topical area (Communications, Tactics Individual, etc.). Identify the task number and then the comment. For example: "Item 1 - radio messages were scratchy, broken, and hard to understand."

3. Additional remarks may be provided in the "Remarks" section on the last page of this data collection sheet.

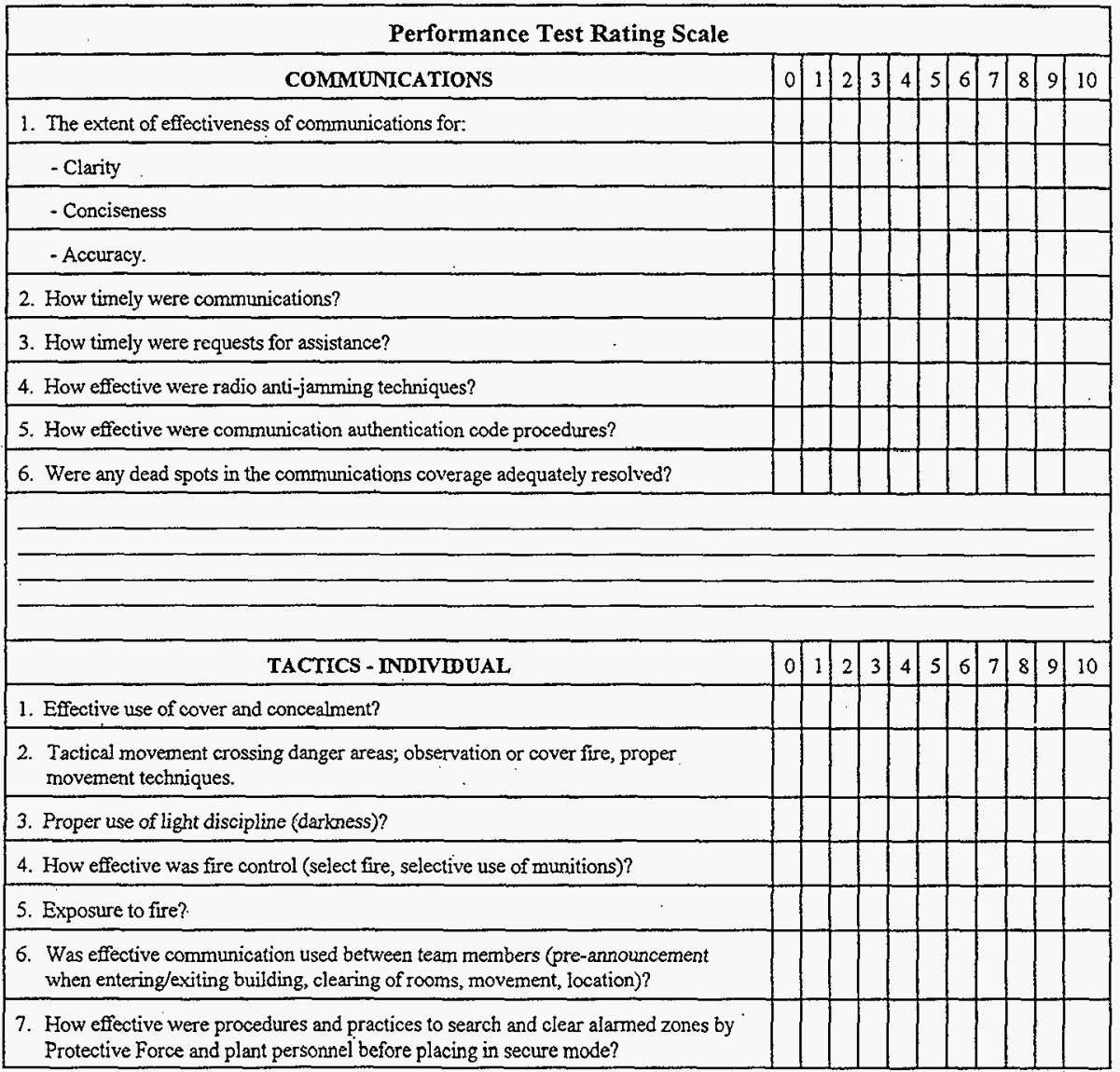




\section{Performance Test Rating Scale}

\section{TACTICS - INDIVIDUAL (cont)}

\begin{tabular}{|l|l|l|l|l|l|l|l|l|l|l|}
\hline 0 & 1 & 2 & 3 & 4 & 5 & 6 & 7 & 8 & 9 & 10 \\
\hline
\end{tabular}

8. How effective was Protective Force post coverage (determine through casual observation)?

9. Were smoke and other pyrotechnic devices effectively used?

\section{TACTICS - TEAM}

\section{\begin{tabular}{l|l|l|l|l|l|l|l|l|l|l|}
0 & 1 & 2 & 3 & 4 & 5 & 6 & 7 & 8 & 9 & 10 \\
\hline
\end{tabular}}

1. Effective use of cover and concealment?

2. Proper deployment from vẹhicle?

3. Alternate routes planned and used?

4. Effective team movement techniques (cover, supporting fire, coordinated movement, spacing)?

5. Effective use of tactical vehicles (blocking, cover)?

6. How effective were command, control, and coordination?

\section{RESPONSE AND CONTAINMENT}

\begin{tabular}{l|l|l|l|l|l|l|l|l|l|l|}
0 & 1 & 2 & 3 & 4 & 5 & 6 & 7 & 8 & 9 & 10 \\
\hline
\end{tabular}

1. How timely was the response?

2. Effectiveness of internal containment?

3. Effectiveness of extemal containment?

4. Awareness of responding forces as to actions taking place?

5. Use of appropriate forces (number, equipment, training)?

6. Timely notification of outside law enforcement agencies (if appropriate)? 


\section{Performance Test Rating Scale}

\section{APPLICATION OF FORCE}

\begin{tabular}{l|l|l|l|l|l|l|l|l|l|l|}
0 & 1 & 2 & 3 & 4 & 5 & 6 & 7 & 8 & 9 & 10
\end{tabular}

1. Minimum justified force used to prevent entry to plant?

2. Minimum force used to prevent escape of adversary?

\section{EQUTPMENT}

\begin{tabular}{l|l|l|l|l|l|l|l|l|l|l|}
0 & 1 & 2 & 3 & 4 & 5 & 6 & 7 & 8 & 9 & 10 \\
\hline
\end{tabular}

1. Effectiveness of communications equipment?

2. How appropriate were response force vehicles for the situation?

3. How appropriate were response force weapons for the situation?

4. Effectiveness of personal protective gear?

5. How appropriate was response force's personal gear for the situation?

\section{COMMAND AND CONTROL}

\begin{tabular}{|l|l|l|l|l|l|l|l|l|l|l|}
\hline 0 & 1 & 2 & 3 & 4 & 5 & 6 & 7 & 8 & 9 & 10 \\
\hline
\end{tabular}

1. How effective was the chain of command; did everyone know who was in charge at all times?

2. Rate the effectiveness of the Central Alarm Station/point-of-contacton-scene commander in:

\section{- Deploying forces}

- Plotting location of forces

- Keeping all posts and patrols updated on situation

- Notification of key personnel

- Maintaining radio discipline. 
FNNE-EP-0927

Remarks:

NAME

DATE 